\title{
A Quantum Signature for the Cosmos
}

\section{Certain galaxy patterns might encode whether the Universe's primordial density fluctuations were quantum or classical in nature.}

\author{
By Christopher Crockett
}

7 he distribution of galaxies in the Universe might trace its roots to tiny primordial density fluctuations that were magnified when the cosmos ballooned roughly $10^{-35} \mathrm{~s}$ after the big bang. Many argue that these fluctuations were quantum in nature, while others think that they could have had a classical origin. And, cosmological observations are-so far-consistent with both stories. Now, researchers propose a new test that could settle this debate.

In the quantum story, the initial density fluctuations arose from spontaneously created particle pairs that were separated by the rapid inflation of space before they could self-annihilate. These pairs could explain observed spatial correlations among pairs of warms spots in the cosmic microwave background as well as among the placements of pairs of galaxy clusters. But such "two-point" correlations could also arise for density fluctuations caused by a classical source of randomness.

To confirm the correlation source, Daniel Green, of the

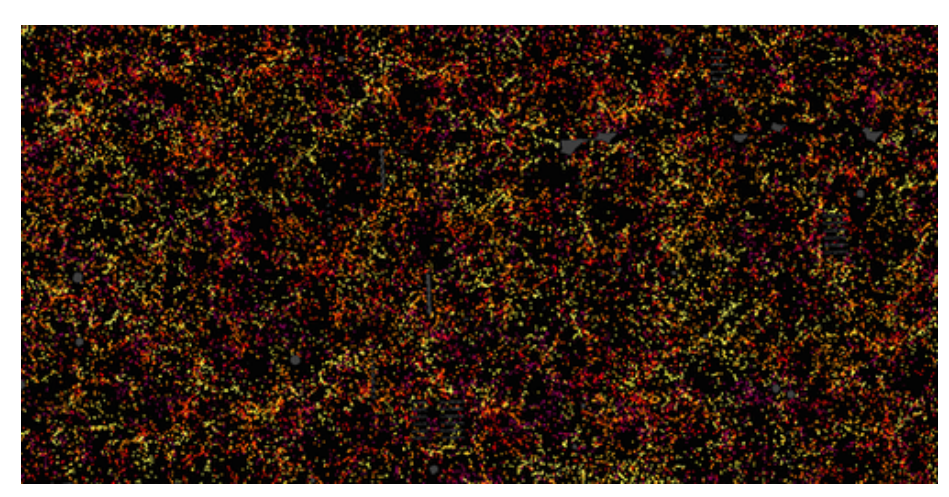

Credit: Sloan Digital Sky Survey
University of California, San Diego, and Rafael Porto, of the German Electron Synchrotron (DESY) in Hamburg, suggest instead looking for certain types of "three-point" correlations. In the quantum case, three particles can sometimes arise out of the vacuum and should lead to three correlated spots in the sky. In the classical case, particle decays during cosmic inflation should also induce three-point correlations but in a different pattern than the quantum scenario.

Finding these patterns could help resolve this classical versus quantum debate. But doing so won't happen overnight. Future galaxy surveys, such as NASA's upcoming SPHEREx mission and ESA's Euclid satellite, will likely help researchers detect three-point correlations, the duo says, though competing sources of correlations, such as those caused by gravity, may take a while to untangle.

This research is published in Physical Review Letters.

Christopher Crockett is a freelance writer based in Arlington, Virginia. 